\title{
Some Inflationary Einstein-Aether Cosmologies
}

\author{
John D. Barrow \\ DAMTP, Centre for Mathematical Sciences, \\ Cambridge University, \\ Wilberforce Rd., \\ Cambridge CB3 0WA \\ UK
}

October 25, 2018

\begin{abstract}
We show how to derive several families of accelerating universe solutions to an Einstein-Aether gravity theory. These solutions provide possible descriptions of inflationary behaviour in the early universe and late-time cosmological acceleration.

PACS numbers 04.50.Kd, 04.20.Fy, 98.80.Cq
\end{abstract}

\section{Introduction}

There has been renewed interest in Lorentz-violating theories of gravity and their consequences for experimental gravity and cosmology. Donnelly and Jacobson [1] have provided a systematic construction of an Einstein-Aether gravity theory of this sort that preserves locality and covariance in the presence of an additional Lorentz-violating ('aether') vector field. This determines a preferred rest frame at each spacetime point, as was also considered by Gasparini [2, and leads to interesting variations on the standard picture for the development of large-scale structure in the universe that a number of authors have examined in detail, see refs [3, 4, 5, 6, 7, 8, 9, 10. The aether vector field, $u_{a}$, and the metric tensor $g_{a b}$ together determine the local spacetime structure. In an isotropic and homogeneous Friedmann universe with expansion scale factor $a(t)$ and comoving proper time $t$, the aether field will be aligned with the cosmic frame and is related to the expansion rate of the universe by

$$
\nabla_{c} u^{b}=\frac{\dot{a}}{a}\left(g_{c b}-u_{c} u_{b}\right)
$$

The Einstein equations are generalised by the contribution of an additional stress tensor for the aether field. If the universe contains a single self-interacting 
scalar field $\phi$, with a self interaction potential $V$ that can now be a function of $\phi$ and the expansion rate $\theta=3 \dot{a} / a$, then the modified stress tensor of Donnelly and Jacobson [1] is

$$
T_{a b}=\nabla_{a} \phi \nabla_{b} \phi-\left(\frac{1}{2} \nabla_{c} \phi \nabla^{c} \phi-V+\theta V_{\theta}\right) g_{a b}
$$

This corresponds to and effective fluid with pressure $p$ and density $\rho$ of the form

$$
T_{a}^{b}=\operatorname{diag}(\rho,-p,-p,-p)
$$

with

$$
\begin{gathered}
\rho=\frac{1}{2} \dot{\phi}^{2}+V-\theta V_{\theta} \\
p=\frac{1}{2} \dot{\phi}^{2}-V+\theta V_{\theta}-\dot{V}_{\theta}
\end{gathered}
$$

with $V(\phi, \theta)$ where $\theta=3 H=3 \dot{a} / a$.

The energy-momentum conservation law,

$$
\dot{\rho}+3 H(\rho+p)=0,
$$

then remains as in general relativity

$$
\ddot{\phi}+3 H \dot{\phi}+V_{\phi}=0
$$

while the Friedmann equation is augmented by the contribution of the aether stress to the energy density $(8 \pi G=1=c)$ :

$$
3 H^{2}=\rho=\frac{1}{2} \dot{\phi}^{2}+V-\theta V_{\theta}-\frac{k}{a^{2}},
$$

where $k$ is the usual Friedmann curvature parameter in the metric (in coordinates $\{t, r, \vartheta, \varphi\})$

$$
d s^{2}=d t^{2}-a^{2}(t)\left\{\frac{d r^{2}}{1-k r^{2}}+r^{2} d \vartheta^{2}+r^{2} \sin ^{2} \vartheta d \varphi^{2}\right\},
$$

We will now set $k$ equal to zero in what follows.

The energy-momentum tensor, and the forms of the density and pressure it contains, are reminiscent of the form required when a simple bulk viscosity is added to a perfect fluid close to equilibrium [11, 12, 13. However, there are differences. The addition of a bulk viscosity $\eta(\rho)$ to a fluid with density $\rho$ and isotropic pressure $p$ is obtained by effecting the transformation

$$
(\rho, p) \rightarrow(\rho, p-\theta \eta)
$$

in the equations (4) and the left-hand equality in (6), so the Friedmann equation of general relativity $\left(3 H^{2}=\rho-k a^{-2}\right)$ is left unaltered but the density conservation changes. By contrast, the introduction of the aether field is effected by the transformation 


$$
(\rho, p) \rightarrow\left(\rho-\theta V_{\theta}, p+\theta V_{\theta}-\dot{V}_{\theta}\right)
$$

which differs from the situation with bulk viscous stresses in an expanding universe unless $V_{\theta}=0$ and $\dot{V}_{\theta}>0$.

\section{Simple exact solutions}

Solutions of these equations are of interest in two cosmological eras. The first is in the early period where accelerated 'inflationary' expansion might occur for a finite time interval, solving the traditional horizon, flatness, and isotropy problems while creating a distinctive inhomogeneity spectrum which leaves its gravitational imprint on the microwave background radiation anisotropy and statistics. The second is at late times when the universal expansion is observed to be accelerating because of the influence of some gravitationally repulsive stress, aka 'dark energy'. It may be important for a viable cosmological model to exhibit both periods of accelerated expansion in order to be consistent with all astronomical observations. It is not clear whether a single scalar field might be responsible for the early and the late-time acceleration and so far there is no compelling cosmological model in which it is.

We look for a general scale invariant solution of (5)-(6) in which

$$
V(\theta, \phi)=V_{0} \exp [-\lambda \phi]+\sum_{r=0}^{n} a_{r} \theta^{r} \exp [(r-2) \lambda \phi / 2],
$$

where $V_{0}, \lambda$ and $\left\{a_{r}\right\}$ are constants. Note that the series could be extended to negative $r$ if required.

This choice of potential subsumes the simple cases with $V(\theta, \phi)=f(\phi) \theta^{2}$ of Kanno and Soda [14 and $V(\theta, \phi)=f\left(\theta^{2}\right)$ of Zlosnik et al 7 considered earlier for specific purposes, but does not include the choice $V(\theta, \phi)=\frac{1}{2} m^{2} \phi^{2}+\mu \theta \phi$ explored in ref [1] in the context of inflationary models where $V$ exhibits a minimum in $\phi$.

There exist exact power-law solutions of (15)-(6) with

$$
\begin{aligned}
\phi & =\frac{2}{\lambda} \ln t, \\
a & =t^{B}, \\
\theta & =3 \frac{\dot{a}}{a}=3 B t^{-1} .
\end{aligned}
$$

With these choices in (7) we have

$$
V(\theta, \phi)=\frac{V_{0}+S_{n}}{t^{2}}
$$


where $S_{n}$ is the finite series of constants:

$$
S_{n} \equiv \sum_{r=0}^{n} a_{r}(3 B)^{r}
$$

We see that

$$
V_{\theta}=\sum_{r=0}^{n} a_{r} \theta^{r-1} r \exp [(r-2) \lambda \phi / 2] \equiv \frac{R_{n}}{t^{2}},
$$

where the finite series,

$$
R_{n} \equiv \sum_{r=0}^{n} r a_{r}(3 B)^{r-1},
$$

is a constant. We note also that

$$
V_{\phi}=-\lambda V_{0} \exp [-\lambda \phi]+\sum_{r=0}^{n} a_{r} \theta^{r}\left(\frac{r-2}{2}\right) \lambda \exp [(r-2) \lambda \phi / 2]=\frac{\lambda}{t^{2}}\left(T_{n}-V_{0}\right),
$$

where the finite series

$$
T_{n}=\sum_{r=0}^{n} a_{r}\left(\frac{r-2}{2}\right)(3 B)^{r}
$$

is a constant.

We see from these definitions that

$$
S_{n}+T_{n}=\frac{3 B R_{n}}{2} .
$$

Substituting these expressions for $V, V_{\theta}$ and $V_{\phi}$ into the eqns. (5)-(6), we can obtain the algebraic constraints needed to determine $B$ in terms of the constants $V_{0}, \lambda$ and $a_{i}$ which specify the potential completely.

From eqn. (6) we obtain

$$
3 B^{2}=\frac{2}{\lambda^{2}}+V_{0}+S_{n}-3 B R_{n}
$$

and from eqn. (5) we have

$$
-\frac{2}{\lambda}+\frac{6 B}{\lambda}-\lambda V_{0}+\lambda T_{n}=0 .
$$

Solving these, using eqn. (13), we find

$$
B=\frac{2}{\lambda^{2}}-\frac{R_{n}}{2}
$$


and

$$
V_{0}=3 B^{2}-\frac{2}{\lambda^{2}}-S_{n}+3 B R_{n}=T_{n}+\frac{2}{\lambda^{2}}(3 B-1) .
$$

We note some interesting special cases. When the $a_{i}$ are all zero, so $R_{n}=$ $S_{n}=T_{n}=0$, the potential is the familiar exponential potential [15, 16, 17] and there is power-law inflationary solution when $\lambda<\sqrt{2}$ :

$$
\begin{aligned}
a_{i} & =0, \forall i: B=2 / \lambda^{2} \geq \frac{1}{3}, \\
V_{0} & =\frac{2}{\lambda^{2}}\left(\frac{6}{\lambda^{2}}-1\right) \geq 0 .
\end{aligned}
$$

In general, we see that we can have power-law inflation so long as

$$
B=\frac{2}{\lambda^{2}}-\frac{R_{n}}{2}>1
$$

It is instructive to look at a particular illustrative example. Suppose that only $a_{2}$ is non-zero and the potential simplifies to

$$
V(\theta, \phi)=V_{0} \exp [-\lambda \phi]+a_{2} \theta^{2}=\frac{V_{0}+S_{n}}{t^{2}},
$$

then

$$
R_{n}=6 B a_{2} ; S_{n}=9 B^{2} a_{2} ; T_{n}=0,
$$

and

$$
\begin{aligned}
B & =\frac{2}{\lambda^{2}\left(1+3 a_{2}\right)}, \\
V_{0} & =\frac{2}{\lambda^{2}}(3 B-1)=B(3 B-1)\left(1+3 a_{2}\right) .
\end{aligned}
$$

Here, we see explicitly the requirement on the coupling parameter $a_{2}$ for inflation to occur. We note that it is possible for $a_{2} \neq 0$ to create inflationary expansion (ie $B>1$ ) in cases where the same value of $\lambda$ would not lead to inflation when the aether field is absent. Similar properties are shared by the general case when all the $a_{i}$ are non-zero.

\section{Further exact solutions}

The general system of equations we have solved also simplifies in ways that permit phase portraits to be created if required. If we differentiate the Friedmann equation we get 


$$
\begin{aligned}
6 H \dot{H} & =\dot{\phi} \ddot{\phi}+V_{\phi} \dot{\phi}+V_{\theta} \dot{\theta}-V_{\theta} \dot{\theta}-\theta \frac{d}{d t}\left(V_{\theta}\right) \\
6 H \dot{H} & =\dot{\phi} \ddot{\phi}+V_{\phi} \dot{\phi}-\theta\left(V_{\theta \phi} \dot{\phi}+V_{\theta \theta} \dot{\theta}\right) \\
2 \dot{H} & =-\dot{\phi}^{2}-\dot{\phi} V_{\theta \phi}-9 H \dot{H} V_{\theta \theta}
\end{aligned}
$$

and

$$
\begin{aligned}
3 H^{2} & =\frac{1}{2} \dot{\phi}^{2}+V-\theta V_{\theta} \\
\ddot{\phi}+3 H \dot{\phi}+V_{\phi} & =0
\end{aligned}
$$

In order to solve the last three equations, suppose $V$ has a general separable form:

$$
V(\theta, \phi)=U(\phi)+\mu f(\theta) g(\phi)
$$

then

$$
\begin{aligned}
3 H^{2} & =\frac{1}{2} \dot{\phi}^{2}+U+\mu g\left(f-\theta f_{\theta}\right) \\
\ddot{\phi}+3 H \dot{\phi}+U_{\phi}+\mu f g_{\phi} & =0
\end{aligned}
$$

There is a family of special solutions for which

$$
f-\theta f_{\theta}=C
$$

and so

$$
f=C+F \theta
$$

with $C, F$ constants.

The case considered by Donnelly and Jacobson in [1 is

$$
\begin{aligned}
U & =\frac{1}{2} m^{2} \phi^{2} \\
f & =M \theta \\
g & =\phi .
\end{aligned}
$$

In the case where (16) holds, we can choose

$$
U(\phi)=U_{0} \exp [-\lambda \phi]
$$

so 


$$
\begin{aligned}
3 H^{2} & =\frac{1}{2} \dot{\phi}^{2}+U_{0} \exp [-\lambda \phi] \\
\ddot{\phi}+3 H \dot{\phi}-\lambda U_{0} \exp [-\lambda \phi]+\mu(C+F \theta) g_{\phi} & =0 \\
2 \dot{H} & =-\dot{\phi}^{2}-\dot{\phi} V_{\theta \phi}-9 H \dot{H} V_{\theta \theta}=-\dot{\phi}^{2}-\dot{\phi} \mu g_{\phi} f_{\theta}-9 H \dot{H} \mu g f_{\theta \theta}
\end{aligned}
$$

But in our special case $f_{\theta \theta}=0$ and $f_{\theta}=F$, so

$$
2 \dot{H}=\dot{\phi}^{2}-\dot{\phi} \mu g_{\phi} F
$$

The recipe for solving this system is to pick $g(\phi)$ then $\phi(t)$; solve for $H(t)$ and hence use $t(\phi)$ to obtain $H(\phi)$ and find the constraint on the constants from the Friedmann equation; see for example [18, 19, 20, for corresponding results using this method in general relativistic cosmologies.

Example 1: Pick

$$
\begin{aligned}
f & =M \theta \\
g & =\phi \\
\phi & =A \ln [\tanh (\lambda t)]
\end{aligned}
$$

so

$$
\begin{gathered}
\exp [\phi / A]=\tanh (\lambda t) \\
\dot{\phi}=2 A \lambda \operatorname{cosech}(2 \lambda t) \\
2 H=2 H_{0}-\mu \phi-\int \dot{\phi}^{2} d t=2 H_{0}-\mu \phi+2 \lambda A^{2} \cosh (\phi / A)
\end{gathered}
$$

Therefore, (if $H_{0}=0$ ), we have

$$
U(\phi)=\frac{3 \mu^{2} \phi^{2}}{4}-3 \lambda \mu A^{2} \phi \cosh (\phi / A)+\lambda^{2} A^{2}\left[\left(3 A^{2}-2\right) \cosh ^{2}(\phi / A)+2\right]
$$

Or, asymptotically, keeping $H_{0} \neq 0$, as $t \rightarrow \infty$

$$
H \rightarrow H_{0}+\lambda A^{2}
$$

and $\mu$ has no effect.

Example 2

$$
\begin{aligned}
\phi & =A \operatorname{cosech}(\lambda t) \\
H & =H_{0}-\frac{A \mu}{2 \sinh (\lambda t)}+\frac{\lambda A^{2}}{6} \operatorname{coth}^{3}(\lambda t) \\
a & =a_{0} e^{H_{0} t}[\tanh (\lambda t / 2)]^{-\frac{A \mu}{2 \lambda}}[\sinh (\lambda t)]^{\frac{A^{2}}{6}} \exp \left[-\frac{A^{2}}{12} \operatorname{coth}^{2}(\lambda t)\right]
\end{aligned}
$$


and as $t \rightarrow \infty$ we have

$$
a \rightarrow a_{0} \exp \left[H_{0} t+\frac{\lambda A^{2} t}{6}\right]
$$

Again, $\mu$ has no effect on the inflation.

\section{Example 3}

If we change the time variable to $\tau$, where

$$
d / d t=V^{1 / 2} d / d \tau
$$

and denote $d / d \tau$ by ${ }^{\prime}$, and put

$$
a(t)=\exp [\alpha(\tau)]
$$

then

$$
\begin{aligned}
\phi^{\prime \prime}+\frac{V_{\phi}}{2 V} \phi^{\prime 2}+\frac{V_{\phi}}{V} & =0 \\
3 \alpha^{\prime 2} & =\frac{1}{2} \phi^{2}+1-\frac{3 V_{\theta}}{2 V^{1 / 2}} \alpha^{\prime}
\end{aligned}
$$

and we have an autonomous system when

$$
\frac{V_{\phi}}{V}=-\lambda=\text { constant }
$$

and

$$
\frac{V_{\theta}}{V^{1 / 2}}=\mu=\text { constant }
$$

This system can be explored by standard phase plane techniques although we shall not do that here. These constraints (19)-(20) are satisfied by the choice

$$
V(\theta, \phi)=\frac{\mu^{2}}{4}\left(\theta+\theta_{0}\right)^{2}+V_{0} \exp [-\lambda \phi] .
$$

Setting $\theta_{0}=0$ for simplicity, we see there is a particular exact solution in $t$ time:

$$
\begin{aligned}
a(t) & \propto t^{2 / \lambda^{2}(1+3 \mu)} \\
\phi & =\frac{2}{\lambda} \ln (t) \\
V_{0} & =\frac{2\left[6-\lambda^{2}(1+3 \mu)\right]}{\lambda^{4}(1+3 \mu)}
\end{aligned}
$$

which reduces to the familiar power-law inflation model for an exponential potential when $\mu=0$. However, when $\mu \neq 0$ we see that the aether field has a 
strong effect and enables inflation to occur in situations $\left(\lambda^{2}>2\right)$ where it is impossible in the absence of the aether field. The introduction of $\theta_{0} \neq 0$ for a combination of power-law and exponential expansion.

\section{Discussion}

We have shown how a series of simple ansätze allow exact solutions to be found for Einstein-Aether cosmologies. These models provide exact descriptions of inflationary dynamics in the very early universe or the transition to accelerated expansion at recent cosmological epochs. They show the explicit contribution of the aether field to creating accelerated expansion in situations where inflation would not occur in its absence. They can also be extended to include simple 'tracker' solutions and allow Einstein-Aether theories to be more closely tested by cosmological data sets.

\section{References}

[1] W. Donnelly and T. Jacobson, Phys. Rev. D

[2] M. Gasparini, Phys. Lett. B 163, 84 (1985)

[3] E. A. Lim, Phys. rev. D 71, 063504 (2005), S.M. Carroll and E. A. Lim, Phys. Rev. D 70, 123525 (2004).

[4] B. Li, D. F. Mota and J.D. Barrow, Phys. Rev. D 77, 024032 (2008)

[5] J. A. Zuntz, P. G. Ferreira and T. G. Zlosnik, Phys. Rev. Lett. 101, 261102 (2008).

[6] C. Armendariz-Picon, N .F. Sierra and J. Garriga, arXiv astro-ph 1013.1283

[7] T. G. Zlosnik, P. G. Ferreira and G.D. Starkman, Phys. Rev D 75, 044017 (2007).

[8] X-H. Meng and X-L. Du, arXiv gr-qc1109.0823

[9] M. Nakashima and T. Kobayashi, arXiv: gr-qc 1012.5348

[10] T. Clifton, P. G. Ferreira, A. Padilla, and C. Skordis, arXiv:1106.2476

[11] R. Treciokas and G.F.R. Ellis, Comm. Math. Phys. 23, 1 (1971).

[12] J.D. Barrow, Phys. Lett. B 180, 335 (1986); J.D. Barrow, Nucl. Phys. B 310, 743 (1988).

[13] S. Weinberg, Gravitation and Cosmology, chap. 15, Wiley: New York, (1972). 
[14] S. Kanno and J. Soda, Phys. Rev. D 74, 063505 (2006).

[15] F. Lucchin and S. Mataresse, Phys. Rev. D 32,1316 (1985)

[16] J. J. Halliwell, Phys. Lett. B 185, 341 (1987).

[17] J.D. Barrow, Phys. Lett. B 187, 12 (1987).

[18] J.D. Barrow, Phys. Rev. D 48, 1585 (1993)

[19] J.D. Barrow, Phys. Rev. D 49, 3055 (1994).

[20] J.D. Barrow and P. Parsons, Class. Quantum Grav. 12, 1715 (1995). 